Intersections

Canadian Journal of Music

Revue canadienne de musique
Intersections

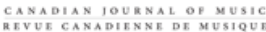

\title{
Jean Molino et les histoires de la musique : de nouveaux modèles pour les réécritures de l'histoire
}

\section{Solenn Hellégouarch}

Volume 31, numéro 1, 2010

URI : https://id.erudit.org/iderudit/1009289ar

DOI : https://doi.org/10.7202/1009289ar

Aller au sommaire du numéro

Éditeur(s)

Canadian University Music Society / Société de musique des universités canadiennes

ISSN

1911-0146 (imprimé)

1918-512X (numérique)

Découvrir la revue

Citer cet article

Hellégouarch, S. (2010). Jean Molino et les histoires de la musique : de nouveaux modèles pour les réécritures de l'histoire. Intersections, 31(1), 163-184. https://doi.org/10.7202/1009289ar
Résumé de l'article

Le singe musicien (2009) réunit quelques textes publiés et inédits écrits par Jean Molino entre 1984 et 2005. Reconnu pour sa théorie de la tripartition, l'auteur y propose de nouvelles bases pour l'appréhension de la sociologie, de l'histoire et de l'anthropologie de la musique. Sa pensée ayant cependant un caractère éclaté, elle nécessite l'élaboration d'une synthèse de ses idées qui constituent de véritables fondements programmatiques. Telle est la tâche que nous nous sommes assignée dans notre étude qui se propose comme un exposé synthétique des propositions de l'auteur quant à l'élaboration du discours historique à partir de quelques textes choisis.
All Rights Reserved (C Canadian University Music Society / Société de musique des universités canadiennes, 2012
Ce document est protégé par la loi sur le droit d'auteur. L'utilisation des services d'Érudit (y compris la reproduction) est assujettie à sa politique d'utilisation que vous pouvez consulter en ligne.

https://apropos.erudit.org/fr/usagers/politique-dutilisation/ 


\section{CHRONIQUE}

Jean Molino et les histoires de la musique : de nouveaux modèles pour les réécritures de l'histoire

\section{Solenn Hellégouarch}

\section{«Pour Une Autre histoire de la MUSiQue»}

En 2006, dans l'édition française du quatrième volume de l'encyclopédie Musiques: Une encyclopédie pour le XXI siècle, dirigée par Jean-Jacques Nattiez, c'est à Jean Molino que revient de clore l'ouvrage avec un essai intitulé : «Pour une autre histoire de la musique : Les réécritures de l'histoire dans la musique du XX ${ }^{\mathrm{e}}$ siècle». Si l'auteur y considère les histoires de la musique du siècle dernier comme des réécritures, il se donne lui-même pour but de «suivre quelques fils du tissu continu de l'histoire tout en proposant [sa] propre réécriture» (Molino 2006, p. 1391). À la différence des encyclopédies qui exposent «l'histoire déjà faite» et «bien éloignée de la réalité de la vie musicale dans toute sa richesse et sa complexité» (ibid., p. 1386), contrairement à ceux qui ont cédé à la pratique historiciste, si Molino propose lui-même « une autre histoire de la musique», ce n'est pas tant pour réinterpréter l'histoire que pour mettre en avant les problèmes méthodologiques liés au discours historique et qui en ont souvent gouverné l'élaboration. À l'aube du nouveau siècle, il semble urgent de repenser le travail historique et de fournir de nouveaux modèles aux réécritures de l'histoire, tâche que Molino semble s'être impartie.

La pensée de l'auteur a ceci de difficile qu'elle est disséminée à travers différents essais et qu'il n'en offre lui-même aucune synthèse. Pour aboutir à un exposé succinct de cette pensée en constellation, il convient de rassembler les idées qui traversent de part en part les textes de Molino et qui, une fois réunies, constituent un véritable programme. Avant de les reprendre une par une et d'identifier les textes dont il sera question, énumérons ces notions pour se faire une première idée : forme symbolique, fait social-symbolique total, individualisme méthodologique, foyer, analyse de situation, histoire virtuelle et contre-factuelle, indétermination historique, vérité, (im)pureté de la musique, sphères / ordres de vie, sociétés restreintes / hiérarchisées, cycles long / court de la communication, ethnographie, animal musicum, phylum, darwinisme universel, descendance avec modification et cladistique. Parmi ces notions, certaines constituent de véritables outils méthodologiques pour la construction du discours historique en musique : principalement, l'individualisme méthodologique, l'analyse de situation, l'histoire virtuelle, l'analyse préalable du niveau neutre, le darwinisme universel et le modèle cladistique. De tels outils, 
souvent issus de disciplines extérieures, permettent de tisser des liens entre les formes de l'histoire présentées par Molino - et c'est pourquoi nous parlerons d'une anthropo-socio-histoire de la musique - , mais aussi de se débarrasser de problèmes méthodologiques qui encombrent le discours historique. Le programme de Molino présente ainsi plusieurs avantages que nous exposerons.

Relativement au discours historique en musique et à ses modèles, nous venons de faire allusion à un texte important paru dans l'encyclopédie Musiques. À cet essai s'ajoute un autre article paru dans la revue Circuit en 1990 et dans lequel l'auteur questionne la notion de «classique» en musique pour définir les «classiques» du siècle dernier ${ }^{1}$. Enfin, c'est en 2009 que paraît le recueil intitulé Le singe musicien : Essais de sémiologie et d'anthropologie de la musique, qui rassemble quelques essais majeurs de l'auteur. Deux d'entre eux ont retenu notre attention : les textes $\mathrm{n}^{\mathrm{os}} 12$, «Pour une sociohistoire de la musique», et 17, «Le singe musicien» — qui a donné son titre à l'ensemble du livre.

Puisque nous croyons que la réflexion de l'auteur trouve son point de départ dans la considération de problèmes méthodologiques fondamentaux, nous les avons réunis dans la première partie de cette étude. Il s'agira donc, successivement, du réductionnisme, de l'holisme et de l'historicisme auxquels Molino oppose les notions de forme symbolique, tripartite, et d'individualisme méthodologique. Parmi les problèmes majeurs soulevés dans les textes s'ajoute la question de la «musique pure» qui n'apparaîtra cependant ici que de façon ponctuelle. La deuxième partie est consacrée aux formes de l'histoire proposées par Molino, principalement l'histoire monumentale, la sociohistoire et l'anthropo-histoire. Nous parlons de forme car nous nous intéresserons principalement aux questions méthodologiques soulevées par les textes et non à des problèmes historiques spécifiques discutés par l'auteur - ce qu'est, par exemple, la musique du XXe siècle selon la réécriture de Molino.

\section{Problèmes fondamentaux}

\section{Réductionnisme et irréductibilité de la forme symbolique}

La critique du réductionnisme est un des fils directeurs de la pensée de Molino. Dans les textes $\mathrm{n}^{\mathrm{os}} 12$ et $17 \mathrm{du}$ Singe musicien, l'auteur s'en prend aux réductionnismes sociologique et anthropologique. Du côté de la sociologie, il s'attaque à la conception marxiste selon laquelle la production musicale est influencée, modelée par les structures socioéconomiques. Il y aurait ainsi un lien direct, déterministe, entre les deux niveaux et selon lequel la musique, et l'ensemble des productions sociales, seraient l'expression de la société elle-même. Parce qu'il est difficile «de mettre en évidence des liens directs entre une œuvre d'art et la société dans laquelle elle a pris naissance» (Molino 2009, p. 265), le sociologue établit des liens entre structures sociales et artistiques en se fondant sur un rapport d'homologie. Quant à l'anthropologie, Molino désapprouve la conception culturaliste désignée par la formule «music as culture» (Merriam 1977) inspirée,

1 Nous ne nous référerons à cet article que de manière ponctuelle, les points essentiels dégagés par notre étude n'étant principalement contenus que dans les trois autres textes discutés. 
entre autres, de l'anthropologue Franz Boas et selon laquelle la musique, produit d'une culture spécifique et unique, est explicable culturellement.

Le problème posé par ces approches est la réduction du phénomène musical à une seule dimension explicative, le social ou le culturel, ce qui ne respecte pas sa complexité, mais surtout sa spécificité : sa qualité de forme symbolique. L'autre problème, et nous y reviendrons, est qu'il y a pour Molino non seulement une nécessité de percevoir le fonctionnement de la musique en tant que forme symbolique avant de la mettre en rapport avec d'autres dimensions, mais aussi de comprendre préalablement l'organisation de la société et de la culture : ensembles complexes, elles sont elles-mêmes constituées de pratiques symboliques, telle que la musique. Au problème du réductionnisme, Molino va ainsi opposer la notion de forme symbolique, selon sa théorie de la tripartition ${ }^{2}$.

Toute production humaine est tripartite et peut être qualifiée de "forme symbolique» : elle est composée de signes qui renvoient à quelque chose, selon la formule de St Augustin. S'appuyant, entre autres, sur les théories de Charles S. Peirce et Gilles-Gaston Granger, Molino précise qu'un tel renvoi est infini : la forme symbolique signifie quelque chose pour quelqu'un qui a dès lors recours à d'autres signes, les interprétants. La forme symbolique est donc tripartite et renvoie à une infinité d'interprétants : c'est ce qui fait, en partie, sa spécificité. Elle peut ainsi donner lieu à de multiples interprétations : le fait musical est total. Il est même un fait social total car, écrit Marcel Mauss (1923-1924) : «ils [les faits sociaux totaux] mettent en branle, dans certains cas, la totalité de la société et de ses institutions» (cité dans Molino 2009, p. 76). Dès lors, le problème posé par les conceptions marxiste, culturaliste et aussi structuraliste, est la réduction de cette faculté de renvoi à une interprétation allégorique et à un sens déterminé. Les conséquences du réductionnisme sont davantage néfastes dès lors que le spécialiste des sciences humaines pense détenir la vérité de l'œuvre: «le sens d'une œuvre humaine serait pour lui la vérité de et sur cette œuvre, que ce sens soit à décrypter dans sa lettre ou dans ses rapports avec la société où elle a été produite. Cette réduction de l'œuvre à une ou plusieurs formes ne respecte pas la structure ontologique des artefacts humains [...]» (ibid., p. 269).

Il convient à présent de considérer la forme symbolique comme une réalité autonome ayant ses propres caractéristiques. En effet, comme le souligne Nattiez, résumant ainsi un texte capital de Molino intitulé «Note sur la situation du symbolique» (1978) : "le symbolique est aussi réel que l'économique, le biologique, le sociopolitique» et «c'est pour cela qu'il faut lui accorder une place spécifique et reconnaître ses propres modalités de fonctionnement aux côtés des trois autres ordres de réalité distingués et distincts les uns des autres »

2 Rappelons que la tripartition est une conception valable pour toute forme de production et d'action humaine. Prenons le cas de la composition d'une ouvre musicale. Cette activité de l'homme laisse une trace matérielle qui est le résultat de stratégies productives et qui donnera lieu à des stratégies réceptives. Il y a donc trois dimensions de l'œuvre, trois niveaux : neutre, poïétique et esthésique. Ces trois niveaux sont autonomes, ou quasi-autonomes, et ne se correspondent pas nécessairement : la communication entre le créateur et le récepteur, entre le poḯtique et l'esthésique, n'est pas parfaite. De ce fait, l'analyse d'une œuvre musicale est triple et ces trois niveaux analytiques peuvent aboutir à des résultats différents. 
(Nattiez dans Molino 2009, p. 19). Le symbolique est alors érigé en véritable outil : ne relevant ni du matériel ni de l'esprit, et parce qu'elle est un produit de l'hominisation, Molino rapproche sa notion de forme symbolique du «monde 3» de Karl Popper (Molino 2009, texte $\mathrm{n}^{0}$ 17, section 17.3.2) ${ }^{3}$. Il révèle ainsi une autre spécificité du symbolique, son caractère constructeur : toute production humaine est une (re)construction symbolique.

$\mathrm{Du}$ fait de sa structure tripartite, de son caractère constructeur et de son autonomie, la forme symbolique ne peut être réduite à l'une de ses dimensions et peut (et doit) être étudiée dans un premier temps en dehors de la société ou de la culture dans laquelle elle est née, avant d'être mise en rapport avec ces deux ensembles complexes. Ce sur quoi Molino insiste, c'est la primauté de l'analyse de niveau neutre - avant l'analyse poḯtique, esthésique, historique, sociale ou culturelle : la musique renvoie à autre chose qu'elle-même, il convient de comprendre d'abord de quoi elle est faite. Il écrit :

Il est vrai qu'il y a autre chose que du son dans la musique, mais le son en constitue évidemment une composante essentielle, et l'on peut parler, en un second sens [nos italiques], de musique pure quand il s'agit d'analyser les propriétés et l'organisation de ce monde sonore. Il est clair que la connaissance de ce son "séparé» ne nous permet pas, à elle seule, de savoir et de comprendre ce qu'est la vie musicale d'une communauté [...], mais elle en constitue un préalable indispensable [id.]. (ibid., p. 276-277)

Enfin, il faut faire la distinction entre deux formes symboliques qui relèvent toutes deux de la tripartition : la musique elle-même et, du côté de l'esthésique mais à un deuxième niveau, le discours sur la musique, le métalangage (ibid., p. 416-418). Au même titre que l'auditeur, l'historien est un récepteur de la musique mais un récepteur particulier, car il possède ses propres stratégies poiétiques qui vont aboutir à l'élaboration d'un discours, une trace qui sera également l'objet de stratégies réceptives : «Les stratégies de réception et d'analyse font venir à l'être des relations imprévisibles : l'histoire est un processus constructif", rappelle Molino (2006, p. 1404). Le discours historique est donc nécessairement multiple : il y a des histoires - et ceci est aussi valable pour l'analyse musicale.

\section{L'individualisme méthodologique, un modèle d'analyse historique et sociologique}

La critique du réductionnisme est à mettre en lien avec celle de l'holisme, qui consiste à élaborer des entités collectives - société, culture, esprit d'époque (Zeitgeist) ou esprit national - considérées comme des ensembles cohérents dans lesquels tout se tiendrait. Or, comme le souligne Molino : «En sociologie comme en histoire, il n'existe pas d'entités collectives bien définies [nos italiques] mais seulement des individus dont les actions entrecroisées font l'histoire

3 Dans la théorie des trois mondes de Popper (1972), le monde 1 est le monde du physique et du biologique, le monde 2, celui du psychique et de la subjectivité. Le monde 3 concerne la connaissance objective, les idées et la culture : produit de l'homme, il n'existe que par rapport à ce dernier mais, distinct des deux autres mondes, il peut revendiquer son autonomie. 
et la société.» (Molino 2009, p. 268) Ainsi se refuse-t-il de concevoir la société et la culture comme des entités homogènes : ensembles complexes de pratiques symboliques, elles résultent des actions et interactions d'individus.

L’auteur en vient donc également à poser le problème des «agrégats et de leur statut", en montrant l'invalidité de la constitution d'agrégats de musiciens ou de données symboliques, des notions de siècle, époque, style, mentalité, Zeitgeist, dont la principale utilité est de nature pédagogique (Molino 2009, p. 269-272). Cela ne revient pas à nier l'existence de macrorégularités, mais au niveau de l'individu ou de l'œuvre, d'une entité singulière et autonome, rien ne garantit que les caractéristiques globales de l'agrégat permettent d'expliquer l'entité individuelle (voir aussi Molino 1990). Pour se débarrasser des entités collectives comme point de départ analytique, et s'inspirant des théories de Max Weber et Popper, Molino propose d'adopter la théorie de l'individualisme méthodologique $^{4}$ (voir Molino 2006, section 1; 2009, texte $\mathrm{n}^{0} 12$, sections 12.1 et 12.2) en partant du constat suivant :

4 «Le social et d'une manière générale les phénomènes sociaux doivent-ils être décrits ou interprétés en termes d' "êtres collectifs" antérieurs aux individus [paradigme holiste]—ou bien faut-il le faire en les rapportant à des comportements individuels et des relations interindividuelles qui constitueraient sinon la réalité humaine exclusive, du moins les données empiriques premières permettant d'en construire des modèles compréhensibles [paradigme individualiste]?» (Laurent 1994, p. 3) Telle est la question inaugurale que se pose Alain Laurent dans un «Que sais-je?» paru en 1994. Épuisé à ce jour, ce petit livre révèle que si la théorie de l'individualisme méthodologique, qui donne son nom au livre entier, est peu connue des musicologues, elle l'est davantage des sociologues: à preuve l'existence de ce document qui fait état d'une littérature foisonnante l'illustrant. L'auteur retrace ainsi l'historique des paradigmes holiste et individualiste représentés d'un côté, par Aristote, Platon, Saint-Simon, Auguste Comte et Durkheim et, de l'autre, par Bernard Mandeville, Adam Smith, Carl Menger, Ludwig von Mises, Friedrich Hayek, Max Weber et Karl Popper, pour n'en citer que quelques-uns.

Pour le premier groupe, les phénomènes sociaux doivent être abordés par «en haut» : les structures sociales, la société - le «Grand Être» (Comte) - sont des «êtres collectifs» autonomes et antérieurs aux individus, des réalités sui generis (Durkheim) qui déterminent l'individu et constituent le point de départ des sciences sociales. Pour le second, l'unité de base sociale est l'individu - et non les "pseudo-touts sociaux» qui relèvent de l'abstraction et de la généralisation - et la compréhension du phénomène social passe par une réduction - non simplificatrice mais enrichissante - de ce dernier à ses éléments constitutifs distincts pour en revenir à l'essentiel et en aborder plus efficacement la complexité. Le paradigme individualiste considère l'individu en tant qu'acteur (Weber), en tant qu'être humain pensant et actif et non «agi» par une instance supérieure, comme le suggère le paradigme holiste. Ce qui prime, c'est l'action individuelle. Mû par des intentions qui lui sont propres, l'individu agissant adopte cependant une attitude ouverte et adaptative en se donnant la possibilité de prendre en compte les intentions et actions d'autrui (intersubjectivité). Le phénomène social résulte donc, souvent non-intentionnellement, de l'agrégation d'intentions et actions individuelles (Menger). Les sciences sociales doivent ainsi reconstituer et comprendre la logique selon laquelle les individus agissent en fonction de leurs intérêts particuliers et toutes ces activités individuelles vont être reliées pour créer des structures complexes et utiles au plus grand nombre. Il convient ainsi de reconstruire des modèles situationnels, de modéliser les phénomènes non-intentionnels (Popper).

L'individualisme méthodologique a donné lieu à de nombreuses critiques et résistances, notamment en raison de ses «tendances à majorer indûment l'importance de certains facteurs (individuels) et à en sous-estimer d'autres (sociétaux)» (ibid., p. 83). Cette «résistance nuancée» va amener certains auteurs à proposer une voie moyenne dépassant le clivage individualisme / holisme (Simmel, Elias, Piaget) et mettant davantage l'accent sur les interactions entre individus : «la société [...] est un "système d'interactions interindividuelles" et le tout y est équivalent à la somme des relations entre individus» (ibid., p. 95-96). De son côté, Edgar Morin insiste sur les inter (ou rétro) actions entre individu(s) et société et adopte une approche en «circuit» de leurs rapports («la boucle indi- 
La société [...] est constituée d'acteurs autonomes qui peuvent être non seulement des individus mais aussi des organisations et des institutions, dans la mesure où ces dernières présentent un degré suffisant d'indépendance structurelle et fonctionnelle. La société n'est donc à chaque instant que la configuration provisoire [nos italiques] des réseaux d'interactions des différents acteurs, acteurs et institutions étant dotés de stabilités et de durées différenciées [id.]. (Molino 2009, p. 268)

Molino introduit par la suite les notions de foyer et d'analyse de situation, empruntées respectivement à l'historien de l'art Jacques Thuillier (2003) et à Popper (1956).

La notion de foyer est importante dans la mesure où, contrairement à la conception holiste, elle prend en compte l'autonomie des acteurs, ainsi que le caractère provisoire de la configuration sociale. L'individu créateur est situé dans un foyer socioculturel où il a reçu sa formation et entretient des rapports avec d'autres acteurs ou foyers : il prend ainsi place dans un lieu donné et à un moment donné. Lorsqu'il s'apprête à créer une œuvre, il est confronté à un problème (composer cette œuvre), à des contraintes (le style, l'effectif instrumental, les directives d'une commande, etc.) et fait face à un état de la musique (passé et présent). Il devra dès lors déterminer les possibilités qui s'offrent à lui pour résoudre son problème, élaborer des choix et prendre des décisions (les stratégies du musicien), processus qui aboutira à la production d'une ouvre singulière. Tel est ce que doit mettre en évidence l'analyse situationnelle : «il s'agit de tenter, de façon hypothétique mais validable, une reconstruction idéalisée de la situation dans laquelle se trouvait l'acteur» (Molino 2006, p. 1390).

Sur la base de l'individualisme méthodologique et de l'analyse de situation, il s'agit donc d'élaborer des modèles individuels et de montrer comment l'œuvre musicale ne peut être le produit direct de la culture ou de la société : elle est avant tout le résultat d'un processus individuel complexe. C'est sur cette nouvelle base que devraient être conçues la sociologie, l'histoire et la sociohistoire de la musique.

\section{L'historicisme et ses conséquences}

La critique de l'historicisme est le leitmotiv de l'article du quatrième volume de l'encyclopédie Musiques. De même que le réductionnisme et l'holisme, la pratique historiciste pose un problème qu'il faut résoudre. En 1956, Popper dénonçait déjà une telle pratique et en donnait la définition suivante :

Qu'il me suffise de dire que j'entends par là une approche des sciences sociales qui fait de la prédiction historique leur principal but, et qui enseigne que ce but peut être atteint si l'on découvre les «rythmes» ou les «modèles», les «lois» ou les «tendances générales» qui sous-entendent les développements historiques. (Popper 1956; nouv. éd. fr. 1988, p. 7)

vidu $\leftrightarrow$ société»). En terminant par l'exposition des idées de Jean-Pierre Dupuy (l'«autotranscendance du social») et Raymond Bourdon, qui fit (re)connaître l'individualisme méthodologique en France, Laurent conclut un véritable survol épistémologique éclairant qui permet de dépasser et nuancer l'opposition trop simple entre les deux méthodes employées en sciences sociales. 
La pratique historiciste interprète donc les œuvres, acteurs et événements en fonction de ce qu'ils annoncent : il ne s'agit pas tant de s'intéresser au compositeur ou à une œuvre pour ce qu'ils sont que de les inscrire dans un sens précis de l'histoire. Ainsi, chaque élément du passé n'est retenu qu'en ce qu'il participe d'un grand projet et ce qui compte le plus est le résultat d'un tel processus.

Molino relève quatre conséquences majeures de l'historicisme et leur oppose quatre arguments :

a) Progrès et linéarité : l'historicisme est évolutionniste et implique la notion de progrès. L'histoire relève ainsi d'un processus linéaire au sens déterminé et l'historien explique l'enchaînement des événements qui constituent le Grand Récit (Molino 2006, section 3). Selon Molino, l'histoire étant écrite du point de vue du présent, il n'y a pas de Grand Récit : celui-ci doit être réécrit et perd dès lors sa nature, car le sens de l'histoire change. Il n'y a pas une histoire, mais des réécritures de l'histoire.

b) Téléologie : s'il y a un grand projet, une finalité de l'histoire, tout doit être interprété en fonction de ce sens et ne sera retenu que ce qui participe audit projet. Mais cette conception téléologique est incompatible avec l'individualisme méthodologique et la notion de forme symbolique. Réductrice, elle fait l'impasse sur une partie du répertoire et des hommes qui font l'histoire et, dès lors, ne rend pas compte de la richesse et de la complexité de l'activité musicale : «Dès que l'on abandonne la conception téléologique de l'histoire, précise Molino, il ne reste plus qu'à apprécier les solutions apportées par chaque musicien selon leur valeur propre.» (ibid., p. 1401)

c) Jugement de valeur: ce qui n'entre pas dans le sens de l'histoire, la marche du progrès, est inutile car dénué de sens. Molino souligne la confusion entre les notions de progrès et de valeur esthétique :

Trop souvent les histoires de la langue musicale sont des histoires du progrès technique qui se présentent en même temps comme des histoires du progrès esthétique, ce qui explique l'emploi fréquent du qualificatif laudatif «progressiste» pour désigner les créateurs qui ont élargi par leurs innovations le langage de leur temps. (ibid., p. 1430)

Dès lors, ce qui est «progressiste» est "esthétique», au sens premier du terme, et «on ne peut faire de la bonne musique que si l'on va dans le sens de l'histoire» (ibid., p. 1395). Or, progrès et esthétique n'ont rien à voir : «On ne peut [...] utiliser la notion de progrès dans le domaine artistique que dans la mesure où "il se limite strictement à la détermination des moyens techniques dont une volonté artistique se sert dans une intention bien déterminée et bien arrêtée" » (ibid., p. 1430-1431). Progrès technique n'est pas synonyme de chef-d'œuvre. En outre, le jugement esthétique est une donnée variable : «Il est certain d'un côté que les goûts varient et qu'il n'y a pas de tribunal de l'histoire, pas de jugement dernier qui conduise à une hiérarchie définitive des valeurs.» (ibid., p. 1431) D’où les réécritures de l'histoire, la redécouverte ou la disparition de certains noms.

d) Vérité : parce qu'il y a un sens de l'histoire, il y a donc vérité; et parce qu'il détermine le sens de l'histoire et l'explique, l'historien est le détenteur de 
la vérité de la musique. Mais étant donné la nature symbolique de la musique et de l'histoire, il ne saurait y avoir un sens de l'histoire, une interprétation du fait musical : rien ne certifie que l'historien détienne la vérité.

Ces nouvelles bases méthodologiques posées, il convient à présent d'en identifier et commenter les cadres d'application : l'histoire monumentale, la sociohistoire et l'anthropo-histoire de la musique.

\section{LES FORMES DE L'HISTOIRE SELON MOLINO}

\section{Des réécritures de l'histoire à l'histoire monumentale}

Il n'existe donc pas une mais des histoires, ou plutôt des réécritures de l'histoire : «Et cette chaîne de réécritures, écrit Molino, repose sur un processus plus fondamental : les hommes changent et se trouvent à chaque instant dans une situation nouvelle qui implique une nouvelle vision de l'histoire.» (Molino 2006, p. 1386) Il ne s'agit donc pas de considérer les histoires écrites par nos prédécesseurs comme fausses, mais de comprendre qu'elles ont été écrites selon le point de vue d'une époque :

De génération en génération, non seulement les hommes et le cadre changent, mais, pour en rester au XXe siècle, en 1920, on ne voit plus 1900 comme le voyaient les contemporains, et des transformations analogues se produisent lorsqu'on passe de 1920 à 1950, puis à 2000 : tout le monde, amateurs, critiques et historiens, réécrit l'histoire à partir de son présent. (ibid., p. 1387)

Une telle remarque peut sembler évidente mais elle a le mérite d'inciter l'historien à se débarrasser des préjugés et jeter un autre regard sur le passé, à comprendre pourquoi nos prédécesseurs se sont «trompés » - mais est-ce vraiment le cas? - sans pour autant les rejeter. Dès lors, elle rappelle l'importance de l'analyse situationnelle dans l'élaboration du discours historique et sa validité pour tout acteur de l'histoire : pourquoi tel historien a écrit telle chose? dans quel foyer était-il situé? quel était le problème qu'il devait résoudre? de quelles ressources disposait-il pour y répondre? Telles devraient être les questions à se poser à la lecture de nos prédécesseurs. Cette démarche revient à s'interroger sur les stratégies poiétiques de l'historien - son discours est également une forme symbolique.

L'article de Molino intitulé « Pour une autre histoire de la musique» est une application de l'individualisme méthodologique et une preuve de son efficacité. Ne partant pas des entités collectives holistes mais des individus-acteurs de l'histoire, Molino démontre l'invalidité de la conception téléologique. En réalisant l'analyse de situation de Debussy, des Six et des néo-sériels, par exemple, il démontre qu'il ne saurait exister un sens de l'histoire : ces acteurs étaient confrontés à un même problème - dépasser le cadre de la tonalité - mais ont choisi des solutions qui ne valent pas moins les unes que les autres. Ainsi, l'œuvre de Debussy n'annonce pas celle de Webern, Stockhausen et Boulez, et les Six «n'ont pas apporté de contribution décisive aux transformations de la langue musicale» (ibid., p. 1405). Quant au néo-réalisme, représenté par Boulez, 
Nono et Stockhausen, il devrait être perçu «non comme la réalisation de l'histoire mais comme une véritable aventure» (ibid., p. 1413).

Lanalyse de situation a cette utilité de dégager des problèmes analogues auxquels étaient confrontés différents individus. Ainsi, ce modèle analytique permet de proposer une nouvelle lecture du passé et de se dégager, entre autres, de la conception téléologique : que ce soit Debussy, les premiers Viennois, le groupe des Six, les "néo-classiques», les néo-sériels ou encore Bartók, tous peuvent être envisagés comme des modèles, pour le musicologue ou le compositeur, car considérés pour ce qu'ils sont et dans la situation qui étaient la leur. C'est qu'ils n'ont pas choisi une voie déterminée mais une solution qui leur paraissait la meilleure pour répondre aux problèmes qui leur étaient posés. Ainsi ne devrions-nous pas céder à une histoire de la musique régie par le principe d'opposition, conçue comme la lutte du progrès et de la réaction, du nouveau et de l'ancien : «Elle se présente sous la forme d'un Grand Récit linéaire et orienté [nos italiques] qui conduit, de révolution en révolution, à la musique du présent et annonce même celle de l'avenir.» (ibid., p. 1394) Cette conception, téléologique, ne dégage que deux orientations possibles - oui ou non - et ne respecte pas la multiplicité des possibles, la richesse de l'activité musicale : il n'existe pas une voie royale ou inféconde, mais plusieurs voies possibles.

C'est ce que met en évidence la pratique de l'histoire virtuelle (ibid., section 12). Cette histoire, qui est celle des possibilités, présente deux avantages. Premièrement, en se posant non seulement la question de ce qui s'est réellement passé mais aussi de ce qui aurait pu se passer, elle permet d'identifier les véritables causes d'un fait : il faut donc pratiquer l'histoire contre-factuelle. Deuxièmement, elle permet de "prendre conscience de l'indétermination de l'histoire», ses hasards, et de se débarrasser du «terrorisme historiciste» (ibid., p. 1424) : il n'existe pas de destin providentiel. En outre, la pratique de l'histoire virtuelle, dans le cadre du $\mathrm{XX}^{\mathrm{e}}$ siècle musical, devrait mettre en évidence la coexistence de différents courants et non l'existence d'un courant dominant. Dès lors, quelle serait la forme du discours historique qui rendrait à la fois compte de l'individualité des acteurs de l'histoire, de la coexistence des courants et de la multiplicité des possibles?

Molino reprend la distinction des trois formes d'histoire telles que définies par Nietzsche (1874) : monumentale, traditionaliste et critique (Molino 2006, section 14). Dans Le questionnement moral de Nietzsche (2005), André Stanguennec définit le lien qui unit ces trois formes:

Des trois formes d'histoire servant la vie - «monumentale», «traditionaliste», «critique» — c'est la première qui devrait servir à l'homme créateur et dont les deux autres-point essentiel à souligner-devraient se faire les auxiliaires. L'individu créateur, de même que ceux qui, plus modestement, visent à favoriser sa création, ont besoin de trouver dans le passé des maîtres, des modèles et des encouragements : «lorsqu’un homme qui veut faire de grandes choses a besoin du passé, c'est par le biais de l'histoire monumentale qu'il se l'approprie» [...]. Le monument du passé désigne donc, non l'événement comme fait, mais le monument comme valeur inspiratrice. C'est dans cette mesure que, devant être «sélective» 
et s'obligeant à pratiquer la dure vertu de «justice» vis-à-vis de son passé, l'histoire monumentale met à son service les deux autres. En elle-même, sans la direction que lui imprime l'histoire monumentale, l'histoire traditionaliste fascinerait seulement les esprits voués à la passivité : «elle ne sait en effet que conserver l'histoire, non pas l'engendrer : c'est pourquoi elle sous-estime toujours ce qui est en gestation» [...]. L'histoire traditionaliste peut cependant être utile aux créateurs, parce qu'elle leur fournit une réserve disponible en maîtres, en modèles, en affects, où puiser de façon sélective. C'est à cette sélection que servira aussi l'histoire "critique": "d'où le besoin, d'une troisième façon, la façon critique, et ce, encore une fois, au service de la vie» [...]. Il faut en effet juger le passé, y distinguer ce qui était créateur de ce qui ne l'était pas, conserver ce qui peut rester un modèle, et éliminer sans pitié ce qui n'était que stérilité et faiblesse. Telle est la vertu de justice d'une histoire critique. L'histoire monumentale exige donc les deux autres formes qui sont des organes servant la vie. (Stanguennec 2005, p. 28-29)

Si Molino plaide en faveur de l'histoire monumentale, c'est parce qu'elle est celle des modèles, retenus seulement pour leur valeur propre, «inspiratrice» : «hommes et œuvres [y] entretiennent un dialogue qui échappe au temps de la chronologie et s'inscrit dans un éternel présent en instaurant une "République des génies" qui reprend le thème de la République des lettres de l'âge classique " (Molino 2006, p. 1428). C’est ce que révèle sa ténacité à défendre activement l'individualisme méthodologique et l'analyse de situation, qui formeraient dès lors le cadre analytique de l'histoire monumentale : nous serions ainsi débarrassés de la perspective historiciste et d'une conception historique fondée sur les oppositions, les luttes, pour ne retenir finalement que les œuvres et les acteurs pour ce qu'ils sont.

Comme le souligne Molino, l'histoire monumentale serait la seule capable de faire coexister les acteurs et œuvres a priori incompatibles : "Il ne reste que des œuvres que l'on peut aimer ou ne pas aimer, mais qui n'ont plus à être situées dans l'histoire.» (ibid., p. 1429) Ceci est d'autant plus important dans la situation actuelle, du trop-plein musical, où l'on doit "choisir au milieu de la multiplicité des possibles» et où nous sommes "condamnés à être libres» (Molino 2009, p. 400). Parce que nous avons peut-être été trop dominés durant le $\mathrm{XX}^{\mathrm{e}}$ siècle par l'histoire critique, commandée par la recherche incessante de la nouveauté, et que l'histoire a été écrite comme la lutte du progrès et de la réaction, parce qu'il ne semblait n'y avoir que deux choix possibles (oui ou non), parce que l'atonalité et les deux âges du sérialisme n’ont pas été considérés comme des aventures, mais comme les réalisations d'un sens de l'histoire, et parce que le $\mathrm{XX}^{\mathrm{e}}$ siècle n'a pas été considéré comme les «cents fleurs d'une exploration du monde sonore sous toutes ses formes et dans toutes ses dimensions» (ibid., p. 399), la richesse du monde musical nous a "éclaté» à la figure. Et nous voici bien démunis devant cette multiplicité, sans savoir quels sont les vrais modèles qui devraient nous inspirer et pourquoi ils le devraient. Comme l'histoire monumentale, l'analyse devrait fournir des modèles, sources d'inspiration pour les compositeurs et, à l'ère postmoderne, nous devrions y retrouver cette coexistence d'éléments jugés incompatibles jusqu'ici : «langages et 
techniques échappent désormais aux querelles sectaires et s'offrent à nous dans le même présent de l'histoire monumentale, tous au même niveau et tous disponibles pour les usages, bons ou mauvais, que nous voudrons bien en faire» (Molino 2006, p. 1433).

À l'éclatement historique et des langages musicaux correspond celui de la musicologie et l'apparition de nouvelles disciplines qui obligent à questionner autrement le fait musical : «jusque dans les années 1960, écrit Nattiez, on pouvait se permettre d'aborder le fait musical du seul point de vue de l'histoire. Nous savons que cela n'est plus possible, car il faut faire appel à d'autres disciplines et d'autres points de vue pour rendre compte de la musique.» (Nattiez 2003, p. 26) Parmi ces disciplines, celles qui nous intéressent dans la suite de cette étude sont la sociologie, l'anthropologie et la biologie.

\section{Pour une sociohistoire de la musique}

Le sociologue recherche les liens qui existent entre musique et société. Or, nous l'avons déjà signalé, Molino remarque qu'il est difficile d'établir de tels liens et que ceux-ci ne peuvent se baser sur une vague homologie des structures, surtout dans le cas de la musique, qui «ne dit rien» (Molino 2009, p. 265). Et c'est parce que la musique ne dit rien que le sociologue et l'historien font intervenir des intermédiaires, en se plaçant dès lors du côté poïétique ou esthésique plutôt que neutre : «[ils] ont à juste titre porté leur attention sur "les mondes de la musique" tels qu'ils se constituent aux divers moments d'une culture", observe Molino (ibid., p. 266). L'erreur serait alors d'utiliser les résultats obtenus pour interpréter l'œuvre, d'expliquer l'interne à l'aide des seules données externes : parce que la musique serait de part en part sociale, le sociologue pense détenir la vérité de l'œuvre par l'observation seule de sa relation avec la société. Dans un ouvrage de sociologie de la musique, note Molino : «il est à chaque instant question d'acteurs et de leur place dans la société, mais on n'entend guère la musique, sauf lorsque le sociologue tente d'établir des liens rapides et superficiels de correspondance ou d'homologie entre formes musicales et groupes sociaux.» (ibid., p. 279-280) Cependant, le niveau neutre devrait être sujet à une analyse qui lui est propre et qui devrait être un préalable à toute autre forme d'approche. Ainsi, au lieu de sombrer dans l'interprétation allégorique, fondée sur une approche holiste et réductrice, Molino propose un cadre analytique fondé sur l'individualisme méthodologique, la notion de forme symbolique, irréductible, et une conception de la société en tant que «réseau d'interactions entre individus» (ibid., p. 275), à caractère provisoire, permettant en outre de considérer la musique dans son présent et non plus en fonction de ce qui a suivi.

S'inspirant de Weber, Molino retient la notion de sphères, ou ordres de vie (Lebensordnungen), de la société. La vie est ainsi constituée d'ensembles de conduites qui se sont isolés pour s'élever au rang de champs autonomes et permettent d'appréhender la société sous un angle donné. Il y aurait donc une «sphère de la musique», autonome mais, précise Molino : «il n'y a pas à en faire la sociologie comme s'il s'agissait de quelque chose qui fût extérieur à la société et qu'il fallait y faire rentrer» (ibid.). Il ne faut cependant pas confondre cette 
autonomisation avec l'idée de purification. En effet, il y a, d'un côté, une autonomisation de la musique par l'apparition, par exemple, du marché musical, de l'édition et bien sûr des sciences de la musique, mais on ne saurait dire qu'il y a eu purification de la musique.

La musique définie comme pure, c'est-à-dire coupée de ses fonctions et circonstances et considérée sous ses aspects seulement formels, voire dépourvus d'affects (cf. Dahlhaus 1978), est un phénomène récent et ne concerne pas toutes les sociétés. En outre, selon la conception sociologique, la musique ne peut être pure car elle entretient des rapports avec autre chose qu'elle-même : elle est donc foncièrement impure. Comme le propose Molino, le seul moment où l'on peut parler de musique pure correspond à l'étude du matériau sonore (cf. p. 163). Ceci lui permet de rappeler l'indifférence des sociologues pour le niveau neutre et leur ténacité à penser que la musique est de part en part sociale, mais aussi d'insister sur la connaissance du matériau sonore comme préalable à l'approche musicale. En outre, si les sociologues s'intéressent peu au matériau musical - du moins, de la mauvaise façon —, Molino remarque également le peu d'intérêt des musicologues pour la sociologie : «On en reste ainsi, dans les deux configurations opposées, à la juxtaposition du social et du sonore ou, si l'on veut, à une coexistence qui n'a rien de pacifique entre l'intérieur et l'extérieur.» (Molino 2009, p. 280)

S'il y a confusion sur la place qu'occupe la musique dans la société et sur sa nature, c'est qu'il y a confusion sur la nature même de la société5. Molino distingue deux types de sociétés (ibid., texte $\mathrm{n}^{0} 17$, section 17.4) : les sociétés restreintes, homogènes, celles des chasseurs-cueilleurs, qui sont caractérisées par un cycle court de la communication - le créateur est aussi le récepteur, et inversement, d'où une possible analogie entre stratégies productives et réceptives; et les sociétés hiérarchisées, étatiques, caractérisées par le cycle long de la communication - le créateur et le récepteur sont deux acteurs distincts, la communication entre les deux peut donc être imparfaite et leurs stratégies productives et réceptives peuvent être contradictoires - et l'absence de culture homogène - il y a, au contraire, une dichotomie culturelle sociale et esthétique, deux cultures opposées ${ }^{6}$.

La situation actuelle de la mondialisation instaure un nouveau cycle de communication, mondial, marqué par l'apparition de nouveaux modes de diffusion,

5 Et cette confusion amène à projeter sur les autres sociétés notre propre conception de la musique, nos propres catégories.

6 Selon Molino, cette séparation résulte de l'apparition de l'écriture, autre forme symbolique, qui a entraîné quatre conséquences majeures (Molino 2009, texte $\mathrm{n}^{0}{ }_{17}$, section 17.4.2) : a) la complexification de l'organisation musicale; b) la séparation de la société en deux couches (lettrés et illettrés) et de la musique en deux domaines (théorique et pratique); c) la division esthétique de la musique, avec d'un côté le Grand Art ou l'Art, et de l'autre le non-art; d) la naissance de l'Art pour l'Art, de la conception de "musique pure», qui n'est d'ailleurs pas une invention de la culture européenne : elle est présente dans les autres grandes civilisations (voir l'exemple de l'art qin en Chine dans ibid., p. 438-439). Du reste, Molino montre l'invalidité du mot Art, qui renvoie à des considérations esthétiques : "la musique n'est pas un Art» mais de l'art (ars), un savoir-faire, un «ensemble de moyens réglés en vue d'une fin" (ibid., p. 403). Ceci est d'autant plus vrai à l'ère actuelle de la coexistence des musiques, où il est difficile de dire ce qui serait ou non de l'Art et selon quels critères. 
l'ordinateur et l'enregistrement, grâce auxquels nous sommes confrontés à une «multiplicité de "musiques"»:

tout ce que nous aurions le droit de faire, c'est de respecter leur spécificité et de nous garder surtout de commettre le péché majeur, le péché d'ethnocentrisme, en les séparant de leur contexte d'origine, de ce que les gens éprouvent, pensent et disent lorsqu'ils font ou écoutent de la «musique» (Molino 2009, p. 401-402).

Mais il y a aussi universalité, car si la musique circule dans le monde, c'est qu'«il existe quelque chose comme "la" musique» (ibid., p. 402). Ainsi, chaque culture diffusée devrait être étudiée à une échelle plus large. À cette diffusion de la musique correspond celle des traditions musicologiques :

La musicologie telle que nous l'entendons n'apparaît alors que comme une «ethnothéorie» parmi d'autres, comme l'ethnothéorie européenne. Nous vivons donc dans « un monde d'ethnothéories» (Nettl, 2005, p. 210): il y a maintenant des «tribus» musicologiques et ethnomusicologiques en Chine, au Japon, en Inde, dans les pays arabo-islamiques, en Afrique, en Europe et en Amérique, chacune avec ses traditions et ses perspectives propres. Rien n'est plus urgent que de les confronter (Qureshi, 1999) afin de se donner comme but au moins idéal l'élaboration d'une véritable (ethno)musicologie générale. (ibid., p. 442)

À l'élargissement du fait musical doit donc correspondre un élargissement théorique.

À partir de telles considérations, quel devrait être le programme sociohistorique? Si Molino parle de sociohistoire et non de sociologie, c'est que : «La sociologie ne peut et ne doit pas reprendre les problèmes à zéro en s'interrogeant en général sur les rapports entre société et musique. C'est ici en effet qu'intervient l'histoire - et c'est pourquoi nous préférons parler de sociohistoire de la musique» (ibid., p. 281). Outre son cadre diachronique, l'histoire apporte la possibilité de procéder à l'analyse de situation : elle nous renseigne, lorsque nous étudions la situation d'un individu, sur l'état de la musique auquel le créateur était confronté à l'intérieur de son foyer socioculturel. En outre, ce créateur a son histoire et est soumis à des contraintes purement musicales qui l'amènent à élaborer des choix et prendre une décision : "C'est que le matériau musical n'est pas amorphe : il a une consistance propre et ne peut être organisé qu'en respectant les possibilités ouvertes par ses "logiques intrinsèques" à un moment donné de son évolution.» (ibid.) Les contraintes auxquelles le compositeur est soumis sont sociales, mais aussi techniques, et c'est l'histoire qui permet de rendre compte de ces dernières.

Tel est le fonctionnement de la sociohistoire : considérer d'abord l'individu dans son foyer socioculturel et qui occupe une certaine position dans l'évolution du langage musical, tout en considérant que l'état de la musique, quand il crée, n'est pas un champ statique mais ouvert et dynamique-c'est ce que les

7 La notion de «logique intrinsèque» provient de Music, the Arts, and Ideas de Leonard B. Meyer (1967). 
linguistes appellent la «synchronie dynamique» (ibid., p. 282). C'est à partir de ce champ que le compositeur procédera à une construction : il n'y a pas de style prédéterminé, mais seulement des choix, des parcours individuels, à partir de ce à quoi le compositeur est exposé à son époque et dans sa société-d'où le couplage sociologie-histoire. L'individu créateur enrichira ainsi le champ musical par la création d'une œuvre singulière qui s'inscrira dans l'état de la musique auquel sera confronté un autre compositeur. Le programme proposé par Molino peut dès lors se résumer ainsi :

La sociohistoire de la musique, tout en intégrant les œuvres dans la société, met en évidence ce que nous appellerons, dans un oxymore provocateur, les «logiques imprévisibles» de l'évolution musicale : logiques, parce que les œuvres se réalisent à partir des possibilités offertes à la fois par l'état des techniques et les conditions de la vie musicale; mais imprévisibles, parce que les acteurs, musiciens ou non, créent à chaque instant par leurs décisions une situation nouvelle. (ibid., p. 284)

Molino ajoute une dernière donnée fondamentale à ce programme. Nous savons que si l'on choisit d'étudier les liens qui existent entre musique et société, nous ne devrions pas considérer la société comme une entité globale et homogène. Pour ce faire, le chercheur devra donc passer par l'individualisme méthodologique, mais il devra également adopter une attitude ethnographique, observatrice et descriptive, à la manière d'un La Bruyère, pour donner un portrait fidèle de l'activité musicale, de sa diversité, en tant qu' "activité humaine fondamentale» (ibid., p. 279).

\section{Pour une anthropo-histoire de la musique}

L’anthropologie est la «science qui s’intéresse aux caractéristiques physiques, sociales, politiques, religieuses et culturelles de l'être humain, en le comparant aux animaux, ou en comparant divers peuples ou sociétés humaines» (Antidote $R X v 8$, 2008). Quoique simpliste, cette définition permet de distinguer deux approches anthropologiques chez Molino.

La première est celle que nous venons d'esquisser. La sociohistoire de la musique relève en partie de l'anthropologie, et plus exactement de l'ethnologie, dont l'étape ethnographique est un préalable : elle observe et décrit les différentes pratiques symboliques qui constituent la société ${ }^{8}$. La deuxième approche - celle qui nous intéresse ici - est celle qui distingue l'homme de l'animal et d'où émerge l'animal musicum, le singe musicien. Avant de présenter ce qu'est l'anthropo-histoire de la musique selon Molino, il est indispensable de comprendre les idées qui en constituent le fondement.

Pour appréhender le fait musical anthropologiquement parlant, Molino considère la musique pour elle-même ou en rapport avec d'autres formes symboliques. Dans le second cas, et partant du problème que pose la notion de

8 Précisons que c'est parce que ces pratiques symboliques s'organisent différemment selon les sociétés, parce qu'une société donnée ne forme pas un tout cohérent, que Molino fait la distinction entre société restreinte et société hiérarchisée. 
«musique pure», il questionne le rapport qu'entretient la musique avec deux autres «familles», le rythme et le langage, qu'il définit comme autonomes, mais qui interagissent avec la musique selon un ensemble de réseaux (Molino 2009, texte $\mathrm{n}^{\mathrm{O}} 17$, section 17.2.2). Molino remarque ensuite que musique et langage présentent trois composantes communes : rythmique, mélodique et sémantique - ou "proto-sémantique de nature affective». Ceci est particulièrement manifeste dans les formes intermédiaires de la musique et du langage, telles que la psalmodie et la cantillation. En outre, langage et musique interagissent également avec les arts de la performance et Molino formule dès lors l'hypothèse de leur genèse commune. Parti du problème posé par le concept de «musique pure», il montre ainsi que dès son origine «la musique n'est pas une espèce naturelle» : elle est un mixte (ibid., p. 408). Il ne saurait dès lors y avoir de «musique pure» conçue comme un retour à l'essence de la musique ou le résultat d'une purification : l'évolution musicale est un "processus continu de diversification dans lequel notre musique pure, loin de représenter un aboutissement, n'est qu'une configuration parmi d'autres dans un paysage incessamment renouvelé» (ibid.).

Après avoir défini ce «soubassement anthropologique commun» (Nattiez dans ibid., p. 50) aux trois familles que sont le rythme, la musique et le langage, Molino distingue six fondements propres à la musique (ibid., texte $\mathrm{n}^{0} 17$, section 17.2.3) : les bruits du monde, le rythme ${ }^{9}$ et le geste, la voix, les affects, les instruments et le langage / métalangage, respectivement caractéristiques de l'homo faber et de l'homo loquens ou symbolicus. Le langage permet à l'homme de parler et d'élaborer des théories : l'hominisation a ainsi mené à un nouveau mode d'existant, la forme symbolique. Or, la spécificité de cette dernière réside en partie dans sa faculté de renvoi infini : reprenant Mauss, Molino redéfinit le fait musical en «fait social-symbolique total» (ibid., p. 417). Si ce sont là les fondements anthropologiques de la musique, Nattiez les entrevoit comme d'éventuels universaux (dans ibid., p. 53) : nous commençons dès lors à comprendre pourquoi la circulation mondiale de la musique est possible.

Molino distingue également huit composantes anthropologiques de l'expérience esthétique - d'autres universaux potentiels - identifiées dans le cadre des sociétés restreintes (ibid., texte $\mathrm{n}^{\mathrm{O}}{ }^{17}$, section 17.4.1) : circonstances et fonctions, expérience sensible, diversité des affects, dimension opérative et virtuosité, comparaison et concurrence, commentaires et métalangage, tradition et nouveauté, présence de la Beauté. Ces composantes sont communes aux sociétés hiérarchisées qui, en outre, en présentent deux autres qui leur sont propres : la distinction entre culture d'en haut et culture d'en bas, et leurs musiques respectives, ainsi que celle entre cycle court et cycle long de la communication (voir Nattiez dans ibid., p. 59). Ces données fondamentales exposées, il est possible d'aborder le programme anthropo-historique proposé par Molino.

9 Pour Molino, le rythme est aussi une forme symbolique : il faut, écrit-il, «reconnaître au rythme en tant que tel l'autonomie que nous n'hésitons pas à accorder à la musique» car il constitue « une réalité aussi complexe que celle-ci»; le rythme est "un ensemble de conduites qui font intervenir le corps aussi bien que la société» (Molino 2009, p. 410). 
Son point de départ n'est pas de situer précisément la naissance de la musique - tâche qui serait bien difficile -, mais il avance l'hypothèse de l'apparition simultanée des premières formes de la pratique musicale et de l'activité technique. Du moins, précise-t-il : «il est impossible de penser que les changements dans le domaine visible de la technique n’aient pas été accompagnés de transformations dans le domaine longtemps invisible du symbolique» (Molino 2009, p. 421). En outre, chaque caractère qui définit l'espèce humaine suit sa propre chronologie. De la même manière : «la musique n'est pas une espèce naturelle, elle est constituée de divers modules, de phylums, qui ne sont pas apparus en même temps et qui ont chacun suivi une évolution particulière " (ibid., p. 419). Elle est une construction. Nous venons d'identifier six de ces lignées évolutives propres à la musique - et celles de l'expérience esthétique. Il est intéressant de noter que ces différents phylums - les «logiques propres» (Weber) constitutives de la musique - peuvent être ainsi suivis de façon autonome, en dehors du cadre social et culturel : «Il faut [...] s'intéresser non seulement à l'inscription sociale [...] de la musique mais aussi aux directions multiples et logiques de [son] évolution", précise Molino (ibid., p. 423). Il n'y a d'ailleurs aucune raison de s'arrêter à l'homme pour identifier ces différents phylums car l'animal - et c'est l'objet de la biomusicologie — possède lui-même des formes de pratiques musicales. C'est ce que montre Molino en faisant l'hypothèse de l'apparition du chant chez les singes (ibid., p. 420-421). Pour comprendre la genèse de la musique, il faut donc distinguer ces "phylums asynchroniques» qui la constituent. Ce recours à la notion de phylum, issue de la biologie, est tout aussi utile pour l'histoire car les nouveautés sont souvent la continuation de phylums plus anciens ${ }^{10}$.

Molino fait l'hypothèse d'une genèse commune de la musique, du langage et des arts de la performance. Pour distinguer les phylums qui ont contribué à la constitution de la musique, notre regard doit donc également porter sur les autres formes symboliques avec lesquelles ils interagissent. Molino souligne ainsi que le rythme, le chant (ou la vocalisation), le mime et la danse, à la base du développement de la musique et du langage, jouent un rôle essentiel dans l'hominisation. Ces formes symboliques élémentaires précèdent l'apparition du langage et de la raison, qui ne sont donc plus les seules étapes de l'hominisation : l'homme est faber, loquens, symbolicum, sapiens, animal rationale, mais aussi animal musicum, un singe musicien (ibid., texte $\mathrm{n}^{\mathrm{o}} 17$, section 17.3.1.).

Résumons. Il y a un "soubassement anthropologique commun" (composantes rythmique, mélodique, sémantique) aux formes symboliques (musique, langage, arts de la performance) d'où dérivent les fondements propres à

10 Voir l'exemple de la filiation Marcel Duchamp-Henry Cowell-Harry Partch-John Cage, dans Molino 2006, p. 1416. En biologie, le terme phylum renvoie à la «souche primitive d'où est issue une série généalogique» et par extension un «ensemble constitué par une forme animale ou végétale (espèce ou groupe plus large) et par ses ascendants et/ou descendants supposés». En linguistique, la notion désigne un «ensemble regroupant plusieurs familles de langues». Les familles ainsi formées partagent des caractéristiques permettant de conjecturer une parenté. "Phylum », dans Dictionnaire français en ligne-Larousse, http://www.larousse.fr/dictionnaires/francais/phylum (consulté le 10 mars 2011). 
la musique et dont il conviendrait de suivre l'évolution. C'est ici qu'intervient l'histoire : elle devrait rendre compte de l'évolution de la musique et de ces transformations, de l'apparition, à différents moments, et des trajectoires des différents phylums qui la constituent, la construisent, mais qui appartiennent également à d'autres formes symboliques.

Molino part de la distinction injustifiable, naguère proposée avec insistance, entre sociétés froides et chaudes et fondée sur l'affirmation qu'il n'y aurait que ces dernières, les sociétés modernes, qui connaîtraient le changement et que, dès lors, les sociétés dites froides seraient restées figées dans un état prémusical (Molino 2009, p. 426). C'est qu'il ne faut pas observer les états musicaux des sociétés par comparaison - ce qui amène l'homme moderne à sous-estimer la musique des sociétés plus traditionnelles - mais indépendamment, pour comprendre leur évolution propre, et par rapport à un état antérieur. Nous retrouvons ici le même principe qui est à la base de l'analyse situationnelle.

Afin de rendre compte de cet état antérieur, de l'évolution et des différentes directions de la musique, Molino propose le modèle du «darwinisme universel», tel que défini par Richard Dawkins (1983) et fondé sur le principe de «descendance avec modification» (Platnick et Cameron 1977; voir Molino 2009, p. 427 ${ }^{11}$ : Évolution $=$ réplication + variation + sélection + isolement des populations ${ }^{12}$. Si le modèle a été appliqué à la biologie, Molino distingue l'évolution biologique de l'évolution culturelle, cette dernière étant différente de la première selon trois points : la transmission des caractères acquis de la culture, la présence de directions privilégiées et la liberté de choix des indivi$d u s$. L'évolution biologique doit être vue comme un exemple de descendance avec modification et non comme un modèle d'explication pour toutes les évolutions - c'est l'application de la «règle de Campbell» (ibid., p. 427-428). Mais le modèle du darwinisme universel reste pertinent pour l'élaboration d'une anthropo-histoire de la musique : il y a «apparition d'une innovation (variation) qui se répète, se transmet et se diffuse ou non (sélection et réplication) et qui, dans le cadre d'une communauté, donne naissance à une culture musicale particulière (isolement des populations)» (ibid., p. 429).

Pour rendre compte de cette «descente avec modification» et des directions prises par la musique, Molino propose l'application du modèle de la cladistique, issu de la biologie, qui consiste à classer les êtres vivants selon leur parenté évolutive : "La cladistique travaille sur n'importe quel ensemble de traits qui changent au cours du temps d'une façon non aléatoire, indépendamment du

11 On notera en outre, comme le rappelle Molino, que le modèle utilisé par Darwin dans sa théorie de l'évolution avait déjà été élaboré avant lui dans le domaine des sciences humaines, notamment en linguistique. Il s'agit du «modèle de dérivation historique» selon lequel «tout état s'explique et ne s'explique que par une évolution à partir d'un état antérieur, avec ou sans intervention d'influences extérieures» (Georges Dumézil cité dans Molino 2009, p. 426).

12 Le recours au modèle darwiniste pourrait surprendre, étant donné les applications nauséabondes qu'on lui connaît, mais il convient de rappeler que les différentes idéologies qui s'en sont réclamées pour justifier, par exemple, l'existence de races supérieures, en ont donné une conception erronée et souvent éloignée de sa définition originelle. Si l'une des idées du darwinisme est celle d'un ancêtre commun, comment peut-on avancer la supériorité d'une espèce par rapport à une autre? 
mécanisme ou du processus.» (O'Brien et Lyman (2003) cités dans ibid.). Selon cette méthode, chaque espèce provient de l'autre par transformation. Ne sont considérés, pour former les classes et rendre compte de l'évolution, que les caractères nouveaux, ou dérivés, et partagés, hérités de l'ancêtre. Il faut noter ici que ce qui compte n'est pas tant le déroulement du temps que les relations d'antériorité et de parenté : il s'agit d'un classement logique et non diachronique. Si l'on transpose ce principe au domaine musical, nous retrouvons les «logiques imprévisibles» déjà mentionnées (cf. p. 173) ${ }^{13}$.

Construire l'anthropo-histoire de la musique, c'est donc rendre compte, à partir de la considération des fondements anthropologiques de la musique et de notre nature de singe musicien, des développements multiples de ces fondements, des différents phylums qui constituent le fait musical à certains moments de son évolution et qui peuvent être communs à d'autres formes symboliques.

\section{POUR UNE ANTHROPO-SOCIO-HISTOIRE DE LA MUSIQUE}

Le programme anthropo-historique proposé par Molino offre cinq avantages majeurs. Le premier est qu'il permet de se débarrasser de la notion de progrès : la théorie du changement, telle qu'exposée par Molino, permet de mettre en évidence les transformations et constantes, la diffusion et les emprunts, les directions multiples du fait musical. Il en résulte un abandon du carcan téléologique : il y a une logique de l'évolution, mais aucune providence. Dès lors, délivrée du progrès et de la conception téléologique, l'histoire ne saurait être décrite selon un processus linéaire : il y a une logique de l'évolution mais pas d'ordre prédéterminé. D’ailleurs, il n’existe pas une logique, mais des logiques évolutives, la multiplicité des possibles et non un processus inéluctable. En outre, le recours à la notion de phylum élimine les problèmes posés par la périodisation - peut-être provisoirement.

Selon le modèle du darwinisme universel, il n'existe pas de "grande musique » : issues d'un même ancêtre, de l'animal musicum, des mêmes fondements anthropologiques, toutes les musiques présentent des liens de parenté et se valent toutes. Le programme de Molino vise ainsi à reconnaître la richesse de la pratique musicale. Il n'existe donc pas non plus de "musique pure» au sens traditionnellement accepté : la musique est une construction et résulte de l'interaction de différentes composantes communes à d'autres formes symboliques et à des moments donnés de son évolution. La musique est un mixte. Il nous faut alors remettre en question nos catégories générales: la cladistique révèlerait sans doute d'autres catégories, de nouvelles filiations, qu'on n'aurait su voir en partant de catégories générales (la coexistence d'éléments incompatibles). Elle remettrait ainsi probablement en cause la validité de certaines catégories

13 Les différentes espèces musicales dérivent logiquement les unes des autres («les traits qui changent au cours du temps de façon non aléatoire»); il y a donc une logique, car le langage musical évolue à partir des possibilités offertes par l'état antérieur des techniques, mais il n'y a pas d'ordre prédéterminé («indépendamment du mécanisme et du processus»); les logiques sont imprévisibles car interviennent également des directions privilégiées et les choix des individus. 
généralement acceptées. Il apparaît ainsi qu'il n'y a pas de définition définitive de la musique : comme la culture et la société, elle est «le résultat provisoire d'une combinaison instable d'éléments hétérogènes» (Molino 2009, p. 425). Enfin, le dernier avantage du programme anthropo-historique de Molino est qu'il encourage l'élaboration d'un discours historique qui ne se fonde plus sur des «constructions arbitraires» :

On a donc besoin [...] d'une véritable histoire «technique» de la musique telle que la concevait Max Weber (1921), ou plutôt d'une chronique (O’Hara, 1988) : il s'agit de suivre, indépendamment de toute mise en relation avec un compositeur ou une époque, le développement de chaque élément du langage musical en tenant compte des lieux, des milieux, des niveaux et des genres [...]. [...] Il ne s'agit pas de définir une pratique commune [...] mais au contraire de retrouver la diversité des modèles et des solutions qui s'offraient à chaque créateur. (Molino 2009, p. 431-432)

On en revient donc à l'analyse de situation et à l'histoire des modèles. L'étape suivante consiste ainsi à "passer de cette histoire conçue comme chronique des techniques à une histoire stratégique, qui en constitue le nécessaire complément» (ibid., p. 433) : ce n'est qu’après avoir défini un état de la musique que la création individuelle peut être abordée.

Dès lors, le programme anthropo-historique apparaît comme une base solide pour un programme de plus grande envergure. Si nous avons choisi de terminer par l'anthropo-histoire de la musique, c'est que nous y voyons une prémisse de l'histoire monumentale et de la sociohistoire. En outre, elle nous apprend que si nous considérions d'abord la musique d'un point de vue anthropologique et en adoptant une attitude plus ethnographique, nous nous débarrasserions des notions et concepts hérités de la tradition historiographique qui nous empêchent de voir ce qu'est réellement la musique et sa diversité. Ce qu'ont de commun les trois formes de l'histoire proposées par Molino est bien la considération des hommes et des œuvres pour ce qu'ils sont, dans un perpétuel présent, dans la cristallisation provisoire de la société ou dans les différents cheminements de l'évolution musicale. De même que Nietzsche considère les histoires traditionaliste et critique comme les auxiliaires de l'histoire monumentale, nous croyons qu'il faudrait considérer l'anthropo-histoire et la sociohistoire comme les bras droits de l'histoire monumentale : la première, multidirectionnelle, offre un réservoir inépuisable de possibilités, de modèles que la seconde cristallise à certains moments de l'évolution, puis que la dernière réunit selon leur seule valeur inspiratrice et leur intemporalité.

\section{RÉFÉRENCES}

Antidote RX $v 8$ : Correcteur, dictionnaires, guides. Logiciel d'aide à la rédaction du français. Montréal : Druide informatique inc., 2008. Article «Anthropologie».

Dahlhaus, Carl. 1978. Die Idee der absoluten Musik. Kassel : Bärenreiter. Traduit de l'allemand par Martin Kaltenecker sous le titre L'Idée de la musique 
absolue : Une esthétique de la musique romantique. Genève : Contrechamps, 1997.

Dawkins, Richard. 1983. "Universal Darwinism». Dans Evolution from Molecules to Man, sous la dir. de D. S. Bendall, 403-425. Cambridge : Cambridge University Press.

Laurent, Alain. 1994. L’individualisme méthodologique. Que sais-je?, $\mathrm{n}^{0} 2906$. Paris : Presses universitaires de France.

Mauss, Marcel. 1923-1924. «Essai sur le don : Forme et raison de l'échange dans les sociétés archaïques». Dans L’Année sociologique, $2^{\mathrm{e}}$ série, tome I. Repris dans Sociologie et anthropologie, 143-279. Paris : Presse universitaires de France, 1950.

Merriam, Alan P. 1977. "Definitions of "Comparative Musicology" and "Ethnomusicology" : An Historical-Theoretical Perspective». Ethnomusicology 21, $\mathrm{n}^{\mathrm{o}} 2$ (mai) : 189-204.

Meyer, Leonard B. 1967. Music, the Arts, and Ideas: Patterns and Predictions in Twentieth-Century Culture. Chicago : Chicago University Press. $2^{\mathrm{e}}$ ed. avec postlude, 1994.

Molino, Jean. 1978. «Note sur la situation du symbolique». Georges Duby, numéro spécial de $L^{\prime} A r c, \mathrm{n}^{\circ} 72: 20-25$. 1990. "Classiques et classicisme à l'âge postmoderne». Circuit 1, $\mathrm{n}^{0} 1$ : 75-81.

2006. «Pour une autre histoire de la musique : Les réécritures de l'histoire dans la musique du XX ${ }^{\mathrm{e}}$ siècle». Dans Musiques. Une encyclopédie pour le XXI siècle, "4. Histoires des musiques européennes", sous la dir. de Jean-Jacques Nattiez, 1386-1440. Arles-Paris : Actes Sud/Cité de la musique. Texte absent de l'édition originale en italien (2004). .2009. Le singe musicien: Essais de sémiologie et d'anthropologie de la musique. Précédé de «Introduction à l'œuvre musicologique de Jean Molino» par Jean-Jacques Nattiez, textes réunis par Jean-Jacques Nattiez en collaboration avec Jonathan Goldman. Arles : Actes Sud/INA. Principaux textes discutés : «Pour une sociohistoire de la musique» (inédit), texte $\mathrm{n}^{\mathrm{o}} 12$ : 265-288; «Le singe musicien» (partiellement inédit), texte $\mathrm{n}^{\mathrm{0}} 17$ : $385-443$.

Nattiez, Jean-Jacques. 2003. "Comment raconter le XX $\mathrm{X}^{\mathrm{e}}$ siècle». Dans Musiques. Une encyclopédie pour le XXI siècle, "1. Musiques du XX ${ }^{\mathrm{e}}$ siècle", sous la dir. de Jean-Jacques Nattiez, 39-67. Arles-Paris : Actes Sud/Cité de la musique. Éd. originale en italien. Il Novecento. Vol. 1, Enciclopedia della musica. Turin : Enaudi, 2001.

2006. "Histoire ou histoires de la musique?». Dans Musiques. Une encyclopédie pour le XXI siècle. "4. Histoires des musiques européennes", sous la dir. de Jean-Jacques Nattiez, 19-49. Arles-Paris : Actes Sud/Cité de la musique. Éd. originale en italien. Storia della musica europa. Vol. 4, Enciclopedia della musica. Turin : Enaudi, 2004.

Nettl, Bruno. 2005. The Study of Ethnomusicology: Thirty-One Issues and Concepts. Urbana-Chicago : University of Illinois Press. 
Nietzsche, Friedrich Wilhelm. 1874. «Vom Nutzen und Nachteil der Historie für das Leben». Dans Unzeitgemässe Betrachtungen, II. Leipzig : C. G. Naumann. Trad. fr., «De l'utilité et des inconvénients de l'histoire pour la vie». Dans Considérations inactuelles I et II, sous la dir. de G. Colli et M. Montinari, 91-169. Paris : Gallimard, 1990.

O'Brien, Michael John, et R. Lee Lyman. 2003. Cladistics and Archeology. Salt Lake City : University of Utah Press.

O'Hara, Robert J. 1988. «Homage to Clio, or Towards an Historical Philosophy for Evolutionary Biology». Systematic Zoology 37, $\mathrm{n}^{0} 2$ : 142-155.

«Phylum». Dans Dictionnaire français en ligne-Larousse, http://www .larousse.fr/dictionnaires/francais/phylum (consulté le 10 mars 2011).

Platnick, Norman I., et H. Don Cameron. 1977. «Cladistic Methods in Textual, Linguistic, and Phylogenetic Analysis». Systematic Zoology 26, $\mathrm{n}^{\mathrm{O}} 4$ (décembre) : 380-385.

Popper, Karl. 1956. Misère de l'historicisme. Traduction d'Hervé Rousseau, révisée et augmentée par Renée Bouveresse, à la demande de Sir Karl Popper. Paris : Plon. Nouvelle éd. française. Agora, ${ }^{\circ} 22$. Paris : Pocket, 1988. . 1972. Objective Knowledge. Oxford : Oxford University Press.

Qureshi, Rebula Burckhardt. 1999. «Other Musicologies : Exploring Issues and Confronting Practice in India ». Rethinking Music, sous la dir. De N. Cook et M. Everist, 311-335. Oxford : Oxford University Press.

Stanguennec, André. 2005. Le questionnement moral de Nietzsche. Philosophie, $\mathrm{n}^{\mathrm{o}}$ 917. Villeneuve d'Ascq : Presses Universitaires du Septentrion.

Thuillier, Jacques. 2003. Théorie générale de l'histoire de l'art. Paris : Odile Jacob.

Weber, Max. 1921. Die rationalen und soziologischen Grundlagen der Musik. Munich : Drei Masken Verlag. Trad. fr., Sociologie de la musique : Les fondements rationnels et sociaux de la musique, Jean Molino et Emmanuel Pedler (dir. et trad.). Paris : Métailié, 1998.

\section{RÉSUMÉ}

Le singe musicien (2009) réunit quelques textes publiés et inédits écrits par Jean Molino entre 1984 et 2005. Reconnu pour sa théorie de la tripartition, l'auteur y propose de nouvelles bases pour l'appréhension de la sociologie, de l'histoire et de l'anthropologie de la musique. Sa pensée ayant cependant un caractère éclaté, elle nécessite l'élaboration d'une synthèse de ses idées qui constituent de véritables fondements programmatiques. Telle est la tâche que nous nous sommes assignée dans notre étude qui se propose comme un exposé synthétique des propositions de l'auteur quant à l'élaboration du discours historique à partir de quelques textes choisis.

\section{ABSTRACT}

Le singe musicien (2009) brings together selected published and unpublished texts written by Jean Molino between 1984 and 2005. Known for his theory of tripartition, Molino proposes a new basis for the understanding of the sociology, history, and 
anthropology of music. The wide-ranging nature of his thinking requires synthesizing his ideas, which constitute true programmatic foundations. This is the task we propose to accomplish in this study: to summarize the author's thoughts on the development of historical discourse from a few selected texts. 